\title{
Outcome of Pancreatic Head Coring in as Key Surgical Treatment in Head Dominant Chronic Pancreatitis in Tertiary Referral Centers of Bangladesh
}

\author{
Rabbi H*a , Rashid MM*a , Hakim HAN ${ }^{\mathrm{b}}$, Ahmed AHMT ${ }^{\mathrm{a}}$, Chowdhury AQ ${ }^{\mathrm{b}}$, Sarder MMㄹ, \\ Raihan HMS ${ }^{\mathrm{b}}$, Nayeem SR ${ }^{\mathrm{a}}$, Ali M ${ }^{\mathrm{a}}$
}

\begin{abstract}
Background: Pancreatic head coring procedure, an organ preserving surgery in which diseased head of pancreas is cored with drainage of main pancreatic duct by lateral pancreatico-jejunostomy. Chronic pancreatitis is a persistently occurring inflammatory disease that causes pancreatic duct stones or calcification, stricture, dilatation of pancreatic duct and irreversible morphological changes resulting intractable pain and losses of exocrine and endocrine function. Appropriate management protocol recognizing underlying pathology, can prevent complications and improves the quality of life. Apart from ductal decompression, in Frey's 'procedure resection of the "pacemaker of pain" (Head of the pancreas) gives excellent outcome.

Methods: In this prospective observational study, we have intended to further validate the Frey's procedure an effective surgical method in head dominant severe chronic pancreatitis. The study was carried out on 106 Bangladeshi patients who underwent Frey's Procedure with Lateral Pancreaticojejunostomy from January 2000 to December 2017.

Result: The study revealed 51 patients (48.11\%) were in 3rd decade of life with 45 male and 61 female. We found 77 patients presented with diabetes mellitus, mostly on insulin. Steatorrhoea was noted in 39 patients and Malnutrition in 79 patients. Visual Analog Scale (VAS) was used for scoring pain. Postoperative morbidity was noted in 29 patients $27.35 \%$ cases, but there was no anastomotic leakage or mortality in the immediate postoperative period in our series.

Conclusion: Quality of life has always been the most important decisive factor for patients with severe chronic Pancreatitis. Pain is the predominant clinical feature and very difficult to control. Correction of diabetes and malnutrition are major challenges too. This study revealed, Frey's procedure with adequate ductal clearance with wide pancreato-jejunal anastomosis in head dominant severe chronic pancreatitis is key to better pain control, improves quality of life and prevents recurrence.
\end{abstract}

Key word: Frey's procedure, chronic pancreatitis, Head coring, pancreatic stone, Lateral Pancreaticojejunostomy, LPJ.

(BIRDEM Med J 2018; 8(2): 151-158)

\section{Author Information}

*Bearing authors share equal responsibility as first authorship

1. Hashim Rabbi, Md. Mamunur Rashid, AHM Tanvir Ahmed, HM Sabbir Raihan, Sarder Rizwan Nayeem \& Mohammad Ali. Dept. of Hepato-biliary-Pancreatic Surgery, BIRDEM General Hospital, Shahbag, Dhaka-1000, Bangladesh

2. H A Nazmul Hakim, Ajmal Quader Chowdhury, Mahmud Mohammad Sarder, Dept. of Surgery, Dhaka Medical College Hospital, Dhaka, Bangladesh

Correspondence: Dr. Hashim Rabbi MBBS FCPS MRCSEd MRCPS (Glasg), Assistant Professor, Dept. of Hepato-Biliary-Pancreatic Surgery, Room 710, BIRDEM General Hospital, 122 Kazi Nazrul Islam Avenue, Shahbag, Dhaka, Bangladesh; email: hrabbi72@gmail.com Received: December 7, 2017 Accepted: February 28, 2018

\section{Introduction}

Pancreatic head coring procedure also an organ preserving procedure in which coring of the diseased head of pancreas done along with MPD drainage. Duodenum preserving pancreatic head resection (DPPHR) is the treatment modality in head dominant chronic severe pancreatitis. Frey's Procedure is widely performed procedure in which coring of pancreatic head along with MPD clearance and drainage by lateral pancreatico-jejunostomy. Pancreatolithiasis incidence is less than 1\% in $\mathrm{CP}$ and $90 \%$ patients have pancreatic stones $^{5}$. It is more common in females ${ }^{8}$. Incidence and 
presentation varies widely in Europe, North American, USA, South African and Asia ${ }^{9}$. It is a disease of remission and relapse, progressive inflammation of pancreas resulting in irreversible destruction and replacement with fibrous tissue ${ }^{1}$. CP is commonly associated with pancreatic ductal stones or calcification, ductal stricture, dilatation with loss of exocrine and endocrine function leading to steatorrhoea, malnutrition and uncontrolled glycemic status ${ }^{1-4}$. Recurrent attacks of intractable pain is the predominant feature. Pancreatic enzymes and analgesics are considered as initial medical therapy. Endotherapy was preferred, if medical therapy fails. Surgery was offered to both medical, endotherapy failure and suspected malignancy ${ }^{6}$. Chronic severe pancreatitis of head is considered "Pacemaker of pain". The patients usually depend on opioid analgesics. Quality of life is the pivotal factor. Besides ductal decompression, coring of pancreatic head in Frey's procedure, gives excellent outcome compared to other procedures concerning quality of life and long term pain relief.

The objective of the study was to further authenticate improvement in quality of life after Frey's procedure in head dominant $\mathrm{CP}$.

\section{Methods}

This retrospective observational study includes 106 patients of head dominant $\mathrm{CP}$, surgically treated with pancreatic head coring Frey's procedure and Lateral Pancreaticojejunostomy from January 2000 to July 2017. Surgeries were performed in BIRDEM General Hospital, Dhaka Medical College Hospital and other hospitals of Dhaka by team of surgeons follow same surgical principle. They were thoroughly evaluated clinically, Visual Analogue Scale VAS for pain scoring and others. Plain x-ray of abdomen (anterior-posterior \& lateral view), Ultrasound scan (USG), Magnetic Resonance Cholangiopancreatogram (MRCP), Computed Tomography scan (CT-scan), Carbohydrate Antigen (CA-19.9), Endoscopic Retrograde Cholangiopancreatogram (ERCP) were instrumental to assess pancreatolithiasis, MPD stricture and dilatation, parenchymal changes like fibrosis, atrophy and mass lesions. Patients were briefed perioperatively about VAS, frequently used scale in health care research to assess pain 11-17. It is marked with 'No Pain' to 'Intolerable or worst pain' at ends and the area inbetween were mild to moderate pain.
Non-surgically treated patients like endotherapy with ERCP were also excluded from this study. Patient requiring Whipple's partial pancreaticoduodenectomy and Beger's procedure were excluded from this study.

\section{Surgical approaches}

Surgery was indicated in unrelenting pain due to extensive stones, dilated MPD, suspicious mass lesion, failure of medical and endoscopic treatment; associated complications. Basic principle of surgery was to remove duct stone(s), stricture(s) as well as the disease segment and wide pancreato-jejunal anastomosis. In Frey's procedure after opening of MPD, removal of calculi and enucleation of the diseased pancreatic head and uncinate process in contiguity with strictured segment of duct of Wirsung. Rim of pancreatic head closed to duodenum was spared along with posterior parenchyma, with or without excising both duct of Wirsung and Santorini ${ }^{12}$. It saves pancreatic neck and preserved posterior capsule of pancreatic head along with body and tail. In both original and modified Frey's procedures, different depths of pancreatic tissue was cored ${ }^{11}$. Second generation ducts stones were also removed. Roux-en-Y Longitudinal/ lateral Pancreaticojejunostomy ensured wide pancreatic drainage. Tissues were histopathologically studied to exclude malignancy.

\section{Post-operative period}

All patients were thoroughly evaluated clinically, VAS and investigations considering objective and subjective complaints. Started oral feeding between $3^{\text {rd }}$ to $5^{\text {th }}$ postoperative days and discharged between $7^{\text {th }}$ to $21^{\text {st }}$ postoperative days. Diabetic patients had more frequent follow-ups and some needed special care. Good glycemic control was maintained.

\section{Post-operative complication}

There are minor and major as well as intra and postoperative complications. Delayed Gastric Emptying (DGE) when nasogastric tube (NGT) required beyond $5^{\text {th }}$ postoperative day, failed to tolerate diet before $7^{\text {th }}$ postoperative day or solid diet before $14^{\text {th }}$ postoperative day. Postoperative Pancreatic Fistula (POPF), when there is clinically significant pancreatic leak with persistent pancreatic fluid drainage or intra-abdominal collection rich in amylase ( $>3$ times serum amylase). Post pancreatectomy Hemorrhage (PPH) was considered as hemorrhage after pancreatic surgery. 


\section{Follow up}

All 106 patients were followed up till July 2017 with at least two outpatient follow-ups. Most patients needed proton pump inhibitor or $\mathrm{H}_{2}$ receptor blocker, pancreatic enzyme supplement.

\section{Results}

This retrospective study of conducted on prospective data of 106 patients of Frey's procedure from January 2000 to December 2017. The patients presented between the ages of 16-67 yrs, revealed 51 patients (48.11\%) were in $3^{\text {rd }}$ decade of life, 29 patients $(27.35 \%)$ between 21-30 yrs age group and 9 patients $(8.49 \%)$ between 41-50 yrs age group. There were 45 male and 61 female. This series shows a slight female predominance $(57.54 \%)$. Females presented at an earlier age than the male patients (Figure 1 and 2).

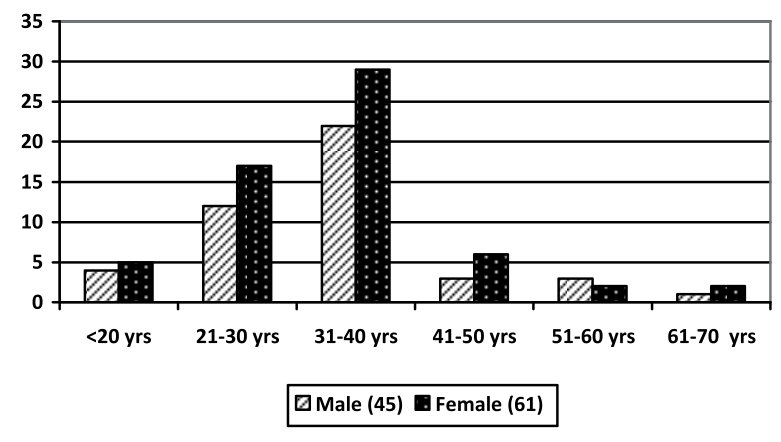

Figure 1. Age distribution of patients $n=106$

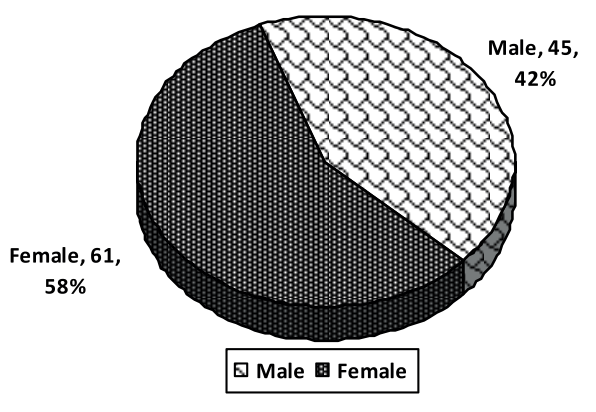

Figure 2. Sex distribution of patient's $n=106$

In our series, we found 77 patients presented with prolong diabetes mellitus, mostly on insulin as our hospital is a tertiary care hospital mostly dealing with diabetic patients. Steatorrhoea was noted in 39 patients and Malnutrition in 79 patients (Figure 3).

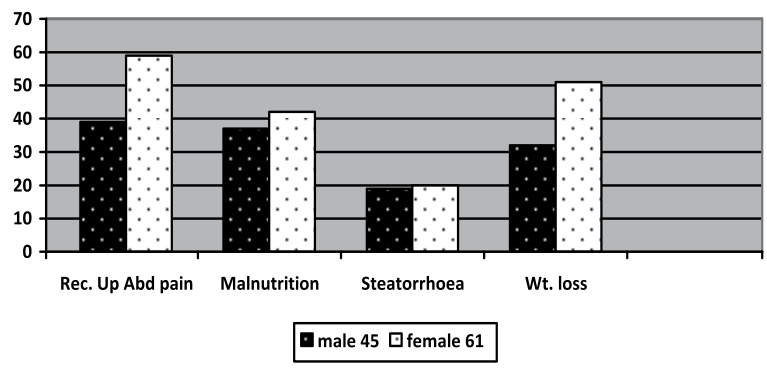

Figure 3. Presenting features of in 138 male and 184 female patients $(n=322)$

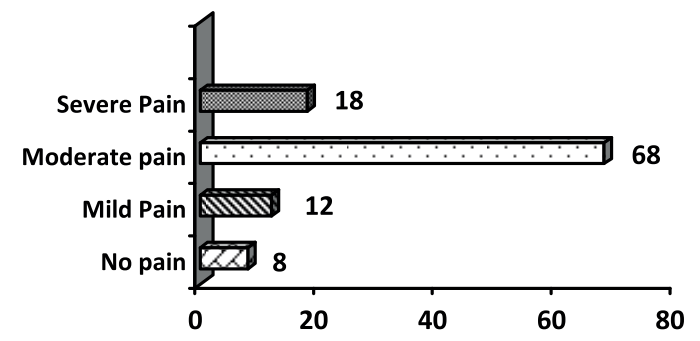

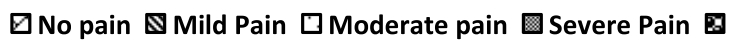

Figure 5. Visual Analog Scale (VAS) for Preoperative assessment of severity of pain

Recurrent abdominal pain of varying intensity was the predominant presenting complaint in almost all patients of CP in our study except 08 patients who presented with malnutrition weight loss and steatorrhoea. Among 106 patients of chronic severe pancreatitis 98 patients presented with recurrent abdominal pain. All patients were briefed about VAS to assess severity of pain, accordingly labeled as mild, moderate and severe pain. About pain $68(64.15 \%)$ with moderate pain not responded to conventional analgesics. About 18 patients $(16.98 \%)$ presented with severe or intolerable pain not responded to any analgesics (figure: 5).

Table I. Preoperative evaluation

Investigation Number of patients

Plain X- ray of abdomen (A-P \& lateral views)

All patients(100\%)

USG of HBP system

Glycemic status

Liver \& Renal function tests

MRCP $78(73.58 \%)$

ERCP $49(46.22 \%)$

CT Scan $\quad 53(50 \%)$

Tumor marker (CA-19.9) $19(17.92 \%)$ 
Plain x-ray of abdomen and US scan of abdomen was the key investigations for diagnosis and to evaluate pancreatic stones and MPD. Although MRCP was invaluable to delineate stone(s), stricture(s), MPD dilatation and parenchymal changes In 78 patients (73.58\%); but in 53 patients (50\%) CT scan was also irreplaceable for planning Frey's procedure. Moreover tumor marker CA 19.9 was done in 19 suspected cases $(17.92 \%)$ with new or severe symptoms or with associated mass lesion. ERCP was performed in 49 patients (46.22\%) (Table I). Among the 23 (21.69\%) had multiple attempts of endoscopic stone extraction and 11 patients $(10.37 \%)$ developed post-ERCP acute pancreatitis. They were treated conservatively and prepared for elective surgery after 4 to 6 weeks or till resolution of symptoms.

In this study, 106 cases of Frey's procedure, revision surgery required for remnant stone in 3 cases of our initial experience (Table II). Three patient underwent hepatico-jejunostomy, in 01 patient for $\mathrm{CP}$ associated lower CBD stricture where Whipple"s Partial Pancreaticoduodenectomy was not possible due to severe adhesion, in 02 patients due to bile duct injury during head coring. Posterior Gastrojejunostomy was done for minor intraoperative duodenal injury In 03 patients. Other additional surgical procedures due to the associated conditions, eg. Cholelithiasis or GB sludge, choledocholithiasis, small peripancreatic abscess were also performed (Table III).

Table II. Additional surgical procedures $n=60$ $(56.60 \%)$ :

\begin{tabular}{lcc}
\hline Name of operation & No. of patients & $\%$ \\
\hline Cholecystectomy & 16 & $15.09 \%$ \\
Choledocholithotomy with & 02 & $1.88 \%$ \\
T- tube & & \\
Hepatico jejunostomy & 03 & $2.83 \%$ \\
Peripancreatic abscess drainage & 05 & $4.71 \%$ \\
$\quad$ Coeliac ganglion block for & 37 & $34.90 \%$ \\
intractable pain & & \\
Gastrojejunostomy & 03 & $2.83 \%$ \\
Revision surgery & 03 & $2.83 \%$ \\
\hline
\end{tabular}

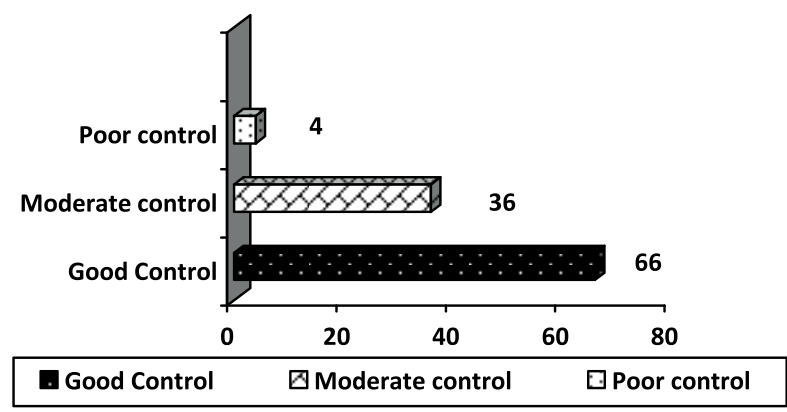

Figure 6. Postoperative assessment of Pain control using Visual Analog Scale (VAS)

Postoperative pain control was a major challenge. In our series we found that good pain relief was achieved in 66 cases $(62.26 \%)$ and 36 patients $(33.96 \%)$ with moderate pain control during immediate postoperative period. However, 04 (3.77\%) patients were complaining of poor pain relief even after surgery (Table 7 ).

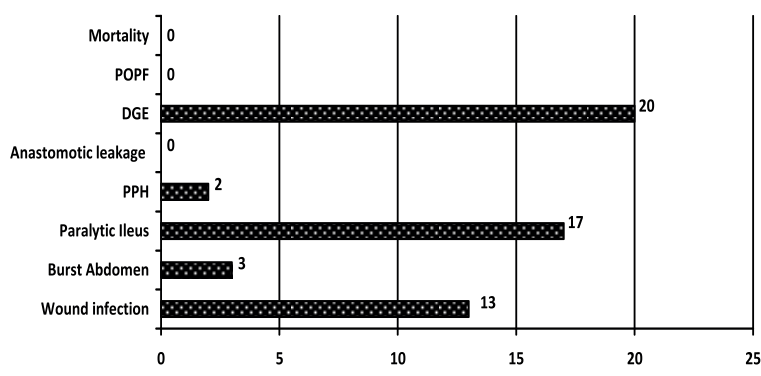

Figure 7. Post-operative complications

Postoperative morbidity was noted in 29 patients 27.35 $\%$ cases, but there was no anastomotic leakage, Postoperative Pancreatic Fistula (POPF) and immediate postoperative mortality or in 30 days in our series. Delayed Gastric Emptying (DGE) noted in 27 patients. Two patients had Post pancreatectomy Hemorrhage (PPH) and required exploration to control hemorrhage. Among them 13 patients had wound infection required wound care, 03 had burst abdomen that required immediate repair with tension suture. Two patients had internal hemorrhage presented with severe anemia, melena and hematemesis postoperative day $7^{\text {th }}$ and $3^{\text {rd }}$ - They were treated conservatively with blood transfusion, somatostatin analogue (Octreotide) but required Laparotomy and hemostatic suture. Among other complications, we found $12(11.32 \%)$ patients with basal atelectasis, $11(10.37 \%)$ with respiratory infections 
and one was MRSA positive and treated accordingly. Seventeen patients (16.03\%) developed paralytic ileus as they were with uncontrolled glycemic status for prolong period and requiring high doses of Insulin and was treated accordingly (Figure 7).

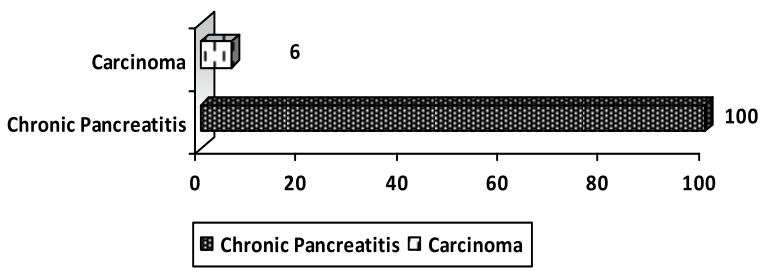

Figure 8. Histopathological study

Multiple samples of pancreatic tissues obtained in Frey's procedure, were sent for histopathological study to exclude malignancy. The study also revealed, 6 cases $(5.66 \%)$ were diagnosed as adenocarcinomas and were treated accordingly. The rest of the 100 patients $(94.33 \%)$ were with chronic pancreatitis (Figure 8).

\section{Discussion}

Chronic pancreatitis is a progressive, recurring inflammatory disorder characterized by irreversible destruction of pancreatic parenchyma and may be associated with disabling chronic pain and permanent loss of exocrine and endocrine function. Duodenum preserving pancreatic head resection (DPPHR) is the cornerstone treatment in head dominant disease. Frey's Procedure is the widely performed organ preserving procedure in which pancreatic head coring, clearance of MPD and drainage by lateral pancreaticojejunostomy. Incidence of CP is increased in fourfold in past 30 years and ranges $3-10$ per 100,000 around the world. Pancreatolithiasis incidence is less than 1\% and common in females. In $90 \%$ cases it is associated with pancreatic stones. ${ }^{5,8}$ It is endemic to tropical countries about 20-125/100,000 persons in South India 4. Chronic pancreatitis carries a mortality 3.6 times higher than individuals without pancreatitis, overall survival rate is $70 \%$ at 10 years and $45 \%$ at 20 years. ${ }^{13}$ Our series patients were randomly selected between the ages of 16-67 yrs, revealed 51 patients $(48.11 \%)$ were in 3rd decade of life, 29 patients $(27.35 \%)$ between 21 30 yrs age group and 9 patients $(8.49 \%)$ between $41-50$ yrs age group. There were 45 male and 61 female. This series shows a slight female predominance (57.54\%) presented earlier than male patients (Fig: $1 \& 2$ ).

Pancreatic pain is usually constant, severe and dull aching in mid-epigastrium often radiates to back, occurs in $80 \%-90 \%$ individuals. ${ }^{12}$ The quality of life is worsened by pain and disease-related complications. Both pancreatic neuritis and pancreatic neuropathy are related with the inflammatory process. ${ }^{12}$ Pain is due to stones or stricture in MPD, resulting in increased intraductal and interstitial pressures. VAS was used to assess the severity of pain, a valid tool of assessment at one point of time as primary or secondary outcomes. ${ }^{17}$ Pancreatic head is considered as "Pacemaker of pain". In our series all had recurrent abdominal pain except 08 patients who presented with malnutrition, weight loss and steatorrhoea. About 68 patients (64.15\%) with moderate pain and 18 patients $(16.98 \%)$ presented with severe or intolerable pain according to VAS (figure: 5). Steatorrhoea reported to be due to dietary fat intolerance with fat intake more than $100 \mathrm{gm} /$ day $^{20}$. In our series, Steatorrhoea was noted in 39 patients (36.79\%) and malnutrition in 79 patients (74.52\%) (Figure 3). Malabsorption responded to pancreatic enzyme supplementation. ${ }^{24,25}$

Pancreatic pathology with calcification leading to diabetes was first published more than 200 years ago by Cawley. Diabetes signifies advanced disease with considerable pancreatic endocrine damage, occurs in $50-70 \%$ of CP and $60-70 \%$ of calcific pancreatitis ${ }^{13}$. World Health Organization (WHO) categorized this as malnutrition-related diabetes mellitus. ${ }^{15}$ We found 77 patients presented with prolong diabetes mellitus, mostly on insulin as our hospital is a tertiary care hospital for diabetic patients. ${ }^{23}$ Uncontrolled glycemic status, recurrent pancreatitis are common. Ketosis resistance is due to defect in ketone body synthesis and/or regulation of counterbalancing hormones. ${ }^{7}$ They need Insulin in gradual dose adjustment or pump. ${ }^{14,15}$

Pancreatolithiasis etiology remains unclear, but malnutrition, dietary cyanogen toxicity, antioxidant deficiency and genetic predisposition are important predisposing factors. Pancreatic juice is saturated with calcium, kept in solution by $\mathrm{HCO} 3$, citrate and pancreatic stone protein (PSP). Alcohol and chronic pancreatitis decreases PSP secretion, causes crystallization and deposition of calcium carbonate 
(CaCO3) to form stones. ${ }^{24}$ Besides, MPD stricture(s) cause stagnation of pancreatic juice and propagates stone formation. Hypercalcemia may also contributes. Radio opaque shadow in plain X-ray locates pancreatic stones. US of abdomen to evaluate stones, MPD and parenchymal changes. ERCP was performed in 49 patients (46.22\%) (Table: 1). Initially, ERCP was a diagnostic tool but precipitates pancreatitis by holding up dye in-between stones and strictured segment. Among them 23 (21.69\%) experienced multiple attempts and 11 patients $(10.37 \%)$ developed post-ERCP pancreatitis. However, ERCP has got the therapeutic role in cases with single stricture and small stones. We found, MRCP was invaluable to delineate stone(s), stricture (s), MPD dilatation and parenchymal changes in 78 patients (73.58\%); CT scan was irreplaceable in 53 patients $(50 \%)$ for planning Frey's procedure. Nutritional correction, control of glycemic status and control of other co-morbidities were key in preoperative preparation. Pancreatic stones can be managed by ERCP, extracorporeal shock wave lithotripsy (ESWL), surgery and denervation ${ }^{24}$. ESWL to fragment large pancreatic calculi then endoscopic clearance of MPD by sphincterotomy and basket swiping for stone removal. Various endoscopic series reported $50-70 \%$ successful MPD clearance and 60-80\% long-term pain relief with $10 \%$ complications rate. The data comparing endoscopic management with surgery is unconvincing. Prospective randomized control trial revealed surgery is superior to endotherapy ${ }^{19}$.

Surgical decompression is a time-honored treatment for long-term pain relief, extensive stones, MPD stricturedilatation, mass lesion, associated complications and failure to medical and endoscopic treatment. ${ }^{10,18}$ Three important arrays were ductal decompression, denervation and pancreatic resection (proximal, distal or total pancreas). The choice of surgery is determined by the extent of disease. ${ }^{11}$ Failure of Partington-Rochelle procedure is the absence of adequate decompression of proximal MPD, uncinate process and second generation ducts ${ }^{12}$. In 1987, Frey and Smith described anterior resection of pancreatic head combining MPD drainage with longitudinal pancreaticojejunostomy. To reduce the risk of entering posterior capsule of head, Frey and Amikura later recommended posterior limit of resection and modified by limiting the pancreatic head coring anterior to MPD. ${ }^{6,7}$ Both original and modified Frey's procedure are safe, but former yields better pain relief. ${ }^{11}$ The Duodenum-preserving pancreatic head resection (DPHR) was proposed by Beger. ${ }^{20}$ Duodenal ischemia, two pancreaticojejunal anastomoses, Postoperative Pancreatic Fistula (POPF, 25\%), Delayed gastric emptying (DGE), ileus are major complications in Beger's procedure (25\%). ${ }^{12}$ Outcome of Partial pancreaticoduodenectomy is equivalent to Frey or Beger's procedure with mortality rate zero.

In our series of 106 Frey's procedure, revision surgery required for remnant stone in 3 cases and in 3 patients hepatico-jejunostomy was done, lower CBD stricture in 01 patient and in 02 patients due to bile duct injury during head coring. In 03 patients posterior Gastrojejunostomy was done for minor intraoperative duodenal injury. Postoperative morbidity was noted in 29 patients $27.35 \%$ cases, but there was no anastomotic leakage, POPF and immediate postoperative mortality or in 30 days in our series. DGE was noted in 27 patients. Among them 13 patients had wound infection required wound care, 03 had burst abdomen that required tension suture. Two patients had internal hemorrhage presented with severe anemia, melena and hematemesis postoperative day $7^{\text {th }}$ and $3^{\text {rd }}$. They were treated with blood transfusion, somatostatin analogue (Octreotide) but required Laparotomy and hemostatic suture.

Pancreatic malignancy is the dreadful sequel of $\mathrm{CP}$ with risk of progression to pancreatic adenocarcinoma $1.8 \%$ after 10 years and $4 \%$ after 20 years. ${ }^{26}$ It often creates a diagnostic dilemma with therapeutic uncertainty. ${ }^{26}$ Although these are commonly chronic inflammatory fibrotic lesions but in $4 \%$ cases associated with pancreatic malignancy and $18 \%$ cases in endemic zone. Patients with TP have a high risk of pancreatic cancer. The risk of developing pancreatic cancer is less in CP. The Risk is higher in hereditary pancreatitis (HP). High index of suspicion with recent weight loss, back pain or jaundice. ${ }^{27,28}$ Multiple samples obtained in Frey's procedure were sent for histopathological study to exclude malignancy. The study also revealed, 6 cases (5.66\%) Incidence of carcinoma in our series and were treated accordingly. However, 100 patients $(94.33 \%)$ were with chronic pancreatitis (Figure 8).

Pain is a decisive factor that hampers quality of life. We found, 98 patients presented with recurrent abdominal pain with moderate pain in 68 patients 
$(64.15 \%)$ and severe or intolerable pain in 18 patients (16.98\%). Postoperative pain control was a major challenge. However, moderate pain control in 36 patients (33.96\%). Literature review revealed $70-80 \%$ pain relief after Frey procedure with improved quality of life. ${ }^{29.33,34}$ In our series, it was with successful outcome in $96.22 \%$ cases with good pain relief in 66 cases $(62.26 \%)$ and only $04(3.77 \%)$ patients were complaining of poor pain relief.

\section{Conclusion}

Pancreatic head coring is an organ-preserving procedure with minimal mortality and morbidity. We found, Surgery is the most effective strategy despite medical and endoscopic management. Control of postoperative pain, diabetes, malnutrition and steatorrhoea is sometimes challenging. We recommend pancreatic head coring surgery as a key procedure in treatment of head dominant chronic pancreatitis, offers better pain control with improved quality of life.

\section{Acknowledgement}

We are thankful to members of Department of Gastrointestinal Hepatobiliary \& Pancreatic Disorders (GHPD), Dept. of Anesthesia and Pain clinic, Dept. of Endocrinology for active participation in the management of these patients.

Conflict of interest: Nothing to declare.

\section{References}

1. Buchler MW, Friess H, Muller MW, Wheatley AM, Beger HG. Randomized trial of duodenum-preserving pancreatic head resection versus pylorus-preserving Whipple in chronic pancreatitis. American Journal of Surgery 1995;169(1):65-69.

2. Friess H, Muller MW, Buchler MW. Duodenum-preserving resection of the head of the pancreas: the future. Digestive Surgery 1994;11(3-6):318-24.

3. Yadav D, Lowenfels AB. The epidemiology of chronic pancreatitis and pancreatic cancer; Gastroenterology. 2013 Jun; 144(6): 1252-61.

4. Lankisch PG, Albert B. Lowenfels; Natural Course of Chronic Pancreatitis; pancreatopedia; Version 1.0, July 27, 2016 [DOI: 10.3998/panc.2016.11]

5. Alberti KG M M, Zimmet PZ. Definition, diagnosis and classification of diabetes mellitus and its complications. Part 1: diagnosis and classification of diabetes mellitus: provisional report of a WHO consultation. Diabet Med 1998; 78:539-53.

6. Mariani A, Bernard JP, Provansal-Cheylan M. Differences of pancreatic stone morphology and content in patients with pancreatic lithiasis. Dig Dis Sci 1991;36:1509-16.
7. Machiki Y, Nimura Y, Kamiya J, Kondo S, Nagino M, Miyachi $\mathrm{M}$, et al. Clinicopathologic study on pancreatic cancer associated with pancreatic stones. Int J Pancreatol 1997; 22:187-91.

8. Khan AKA, Ali L. Tropical calcific pancreatitis and fibrocalculus pancreatic diabetes in Bangladesh. Journal of Gastroenterology and Hepatology, 1997; 12: S48-S52

9. Barman KK, Premalatha G, Mohan V. Tropical chronic pancreatitis. Postgrad Med J 2003; 79:606-15.

10. Chen J, Li NG, Hu Y. The diagnosis and treatment of pancreatic duct stones. Chin J Hepatobiliary Surg 2001;7: 564-65.

11. Tan C, Zhang H, Yang M, Li S, Liu X, Li K. Role of original and modified Frey's procedures in chronic; World $J$ Gastroenterol 2016 December 21; 22(47): 10415-23.

12. Gestic MA, Callejas-Netosa F. Surgical treatment of chronic pancreatitis with frey procedure: current situation. ABCD Arq Bras Cir Dig 2011;24(4):305-11

13. Boonstra AM, Schiphorst HR, Preuper R. Cut-off points for mild, moderate, and severe pain on the visual analogue scale for pain in patients with chronic musculoskeletal pain; Pain 2014; 155: 2545-2550

14. Atsawarungruangkit A, Supot Pongprasobchai. Current understanding of the neuropathophysiology of pain in chronic pancreatitis ; World J Gastrointest Pathophysiol 2015 November 15; 6(4): 193-202

15. Lin YK, Johnston PC, Arce K, Hatipoglu BA. Chronic Pancreatitis and Diabetes Mellitus; Curr Treat Options Gastroenterol 2015 Sep;13(3):319-31

16. Rickelsa MR, Frederico MB, Toledo GS. Detection, evaluation and treatment of diabetes mellitus in chronic pancreatitis: Pancreatology 2013; 13: 336-342

17. Frey CF, Smith GJ. Description and rationale of a new operation for chronic pancreatitis. Pancreas 1987; 2: 701707 [PMID: 3438308]

18. Frey CF, Amikura K. Local resection of the head of the pancreas combined with longitudinal pancreaticojejunostomy in the management of patients with chronic pancreatitis. Ann Surg 1994; 220: 492-504.

19. Sakata N, Egawa S, Motoi F, Goto M, Matsuno S, Katayose Y, Unno M. How much of the pancreatic head should we resect in Frey's procedure? Surg Today 2009; 39: 120-27 [PMID: 19198989 DOI: 10.1007/s00595-008-3816-5].

20. Roch A, Teyssedou J, Mutter D, Marescaux J, Pessaux P. Chronic pancreatitis: A surgical disease? Role of the Frey procedure; World J Gastrointest Surg 2014 July 27; 6(7):| 129-35.

21. Patel HS, Jain SK. Study of prevalence and clinical presentation of fibrocalculous pancreatic diabetes in and around Jabalpur (Madhya Pradesh), Central India; International Journal of Medical Science and Public Health; 2016; 5: 10-20. 
22. Balzano G, Dugnani E, Gandolf A. Effect of Diabetes on Survival after Resection of Pancreatic Adenocarcinoma. A Prospective, Observational Study; PLOS ONE DOI: 10.1371/ journal. pone.0166008 November 4, 2016.

23. Chouduary A, Garg PK, Tandon RK. The role of oxidative stress in tropical pancreatitis and effect of antioxidants supplementation on pain in patients with tropical pancreatitis. J. Gastroenterology and Hepatology 2001; 16: A132(Abstract).

24. Pramod HG, Rakesh KT. Survey on chronic pancreatitis in the Asia-Pacific region. Journal of Gastro enterology \& Hepatology September 2004; 19: 998-1004.

25. Mohan V, Farooq S, Deepa M. Prevalence of fibrocalculous pancreatic diabetes in Chennai in southern India. J Pancreas 2008; 9: 489-92.

26. Choi EK, Lehman GA. Update on Endoscopic Management of Main Pancreatic Duct Stones in Chronic Calcific Pancreatitis; Korean J Intern Med 2012;27:20-29.

27. Chen N, Unnikrishnan IR, Anjana RM, Mohan V, Pitchumoni CS. The complex exocrine-endocrine relationship and secondary diabetes in exocrine pancreatic disorders. J Clin Gastroenterol 2011; 45:850-61.

28. Dana K. A, Andren-Sandberg Al. Pancreatitis - Diabetes Pancreatic Cancer: Summary of an NIDDK-NCI Workshop; Pancreas. 2013 Nov; 42(8): 10.
29. Yadav D, Lowenfels AB . The epidemiology of pancreatitis and pancreatic cancer. Gastroenterology 2013 Jun; 144(6):1252-61.

30. Bhat KRS, Khajanchi M, Prajapati R, Satoskar RR. Evaluation of Pain Preoperatively and Postoperatively in Patients with Chronic Pancreatitis Undergoing Longitudinal Pancreaticojejunostomy; Indian J Surg December 2015; 77:S1098-S1102.

31. Ranjit U, Viswanathan M. Fibrocalculous pancreatic diabetes (FCPD). Acta Diabetologica. 2015; 52: 1-9.

32. Gurusamy KS, Lusuku C, Halkias C, Davidson BR. Duodenum-preserving pancreatic resection versus pancreaticoduodenectomy for chronic pancreatitis. Cochrane Database of Systematic Reviews 2016, Issue 2. Art. No.: CD011521. DOI: 10.1002/ 14651858. CD011521.pub2.

33. Islam SR, Rahman S, Rahman S, Kumar H, Sarkar SA. Outcome of Surgical Drainage of the Pancreatic Duct in Chronic Pancreatitis. Mymensingh Med J 2017 Apr;26: 32734.

34. Rath S, Meher S, Quality of Life after Frey's Procedure in Patients with Chronic Pancreatitis; Journal of Clinical and Diagnostic Research 2016; 10(3): 10-15. 